\title{
Dwarf "transition" types: searching for the holy grail(s)
}

\author{
Patricia M. Knezek ${ }^{1}$, Kate E. Dellenbusch ${ }^{2}$, John S. Gallager, III ${ }^{2}$, \\ and Kenneth R. Sembach ${ }^{3}$ \\ ${ }^{1}$ WIYN Consortium, Inc., 950 N. Cherry Avenue, Tucson, AZ 85719 \\ email: knezek@noao.edu \\ ${ }^{2}$ University of Wisconsin, Dept. of Astronomy, 475 N. Charter Street, Madison, WI, 53706 \\ ${ }^{3}$ Space Telescope Science Institute, 3700 San Martin Dr., Baltimore, MD 21218
}

\begin{abstract}
The evolution of dwarf galaxies, particularly any potential morphological evolution among the different types of dwarf galaxies, remains poorly understood. The interstellar medium (ISM) in dwarf galaxies is thought to be more strongly disturbed by star formation episodes in these low mass galaxies, including supernovae-driven winds during starbursts and star formation induced by galaxy-galaxy interactions. Loss of the ISM has been postulated to result in the evolution of dIrrs to dE/dSphs. However, disentangling the relative importance of environmental (galaxy harassment) versus local stocastic processes on star formation, and their effects on subsequent morphological evolution is still a very active area of research. So-called "transition dwarf" galaxies are galaxies that have properties that fall somewhere between gas-rich dIrrs and gas-poor $\mathrm{dE} / \mathrm{dSphs}$, and may provide clues to the overall evolutionary history of dwarf galaxies. We report on some recent work on a sample of vigorously star-forming dwarf "transition" galaxies, and suggest that they may be examples of the bridge between dIrrs and dEs/dSphs.
\end{abstract}

\section{Introduction}

Dwarf galaxies dominate the population of galaxies by number. As a result, they form a critical component of our overall understanding of how galaxies form and evolve in the universe. Yet many questions about the formation and evolution of dwarf galaxies remain. In particular, one continuing puzzle in galaxy evolution is whether there is an evolutionary connection between the various morphological classes of dwarf galaxies. In particular, are dIrrs and dEs/dSphs related? An extended body of work exists on this topic, both theoretical and observational, and the results indicate that the answer is complex. For example, Knezek, Sembach, \& Gallagher (1999), studied three dwarf galaxies proposed as a sequence of dIrr to "transition" dwarf to dE by Sandage \& Hoffman (1991). They concluded based on the very low star formation rates and the red optical colors of the two galaxies with gas in this sample that they would not evolve into a dE/dSph within a Hubble time. Grebel, Gallagher, \& Harbeck (2003) concentrated on Local Group (LG) dwarfs and determined that while LG dIrrs are unlikely to evolve into LG dSphs based on the fact that dSphs have higher mean stellar metallicities for fixed optical luminosity, the LG "transition dwarfs," whose locus in the luminosity-metallicty diagram is similar to dSphs, are likely progenitors of LG dSphs. Meanwhile, van Zee, Skillman, \& Haynes (2004) observed a sample of $16 \mathrm{dEs}$ in the Virgo cluster and found that rotation is a significant component of the stellar dynamics of some Virgo dwarfs. Furthermore, based on evidence of a relationship between rotation amplitude and galaxy luminosity that agrees with the Tully-Fisher relation, they propose that the dE population in Virgo arises from stripped dIrrs. 
It is likely that the diverse results indicate that there are multiple competing effects. The critical aspect of all proposed evolutionary scenarios for creating gas-poor systems from gas-rich systems, however, is how the gas is removed (e.g., Lin \& Faber 1983, Thuan 1985). This might occur as a result of supernova driven winds during starbursts (Dekel \& Silk 1986, Babul \& Rees 1992, De Young \& Heckman 1994, Spaans \& Norman 1997), or as a result of an external interaction, such as ram pressure stripping (Kormendy 1985, Binggeli 1986), tidal interactions (e.g. Sofue 1994, Mayer et al. 2001 a, b), or galaxy collisions (e.g. Henriksen \& Byrd 1996, Moore, Katz, \& Lake 1996). Disentangling the physical origins of the two main families of low luminosity galaxies, and searching for paths between them remains a difficult task. The potential key to success is the class of "transition" dwarf galaxies, those systems that show a mixture of early and late-type dwarf galaxy characteristics.

\section{What is a "transition" dwarf galaxy?}

Dwarf galaxies come in many flavors, and the classification of dwarf galaxies, like the classification of more massive galaxies, can depend how the author defines the sample. Thus, one interpretation of "transition galaxies" was described by Sandage \& Hoffman (1991) based on their optical and neutral gas composition. The optical morphology is mixed between that of a dwarf irregular and a dwarf spheroidal, while the gas mass to blue luminosity ratio is low for irregular dwarfs, but high for dwarf spheroidals. There is no criteria based on the current star formation rate of the galaxy. Under this scenario, the expectation is that an irregular galaxy undergoes star formation and either exhausts its fuel supply or loses it through supergalactic winds. The galaxy will then slowly fade away. Potential difficulties with this general definition include the fact that dIrrs generally occupy a different place on the metallicity-luminosity relation from dEs, that dIrrs tend to be rotationally supported, while dEs may not be, and that dIrrs generally have low star formation rates, leading to very long gas depletion timescales (often many Hubble times, e.g. Knezek, Sembach, \& Gallagher 1999; van Zee, Haynes, \& Salzer 1997).

A related, but separate interpretation of "transition galaxies" is that of a subsample of blue compact dwarf (BCD) galaxies which have central starbursts but smooth outer optical isophotes, and are relatively gas-poor (van Zee, Salzer, \& Skillman 2001). The idea here is that the galaxies rapidly use and/or lose their gas through the starburst, and will then slowly fade away to a dSph. Potential difficulties with this scenario include the fact that even the lowest $\mathrm{M}_{H I} / \mathrm{L}_{B}$ systems seem to be rotationally supported, and the central starburst remnant will need to fade.

For both interpretations above, the discovery of rotationally supported dEs by van Zee, Skillman, \& Haynes (2004), along with the work of Mayer et al. (2001a,b) showing that repeated tidal shocks experienced by dwarf galaxies that are satellites can both remove the satellites gas and transform it into a dynamically hot system, suggest that what has traditionally been known as the 'angular momentum problem' may not be an issue in all cases. Furthermore, there is a known starburst galaxy, NGC 5253, which is a member of the Centaurus A group of galaxies, and does not appear to be rotationally supported (Caldwell \& Phillips 1989). This suggests, at least for dwarf galaxies in moderate to dense environments, the environmental effects of galaxy harrassment may in fact be enough to turn a dIrr into a dE/dSph. Are there other galaxies like NGC 5253?

In this work, we present a sample of five dwarf galaxies selected to be "transition galaxies" based an optical mix of early and late-type morphological characteristics. The sample and observations are presented in Section 3, the results are discussed in Section 4, and our conclusions are in Section 5. 


\section{Sample and Observations}

We selected six dwarf galaxies that appeared to have a mix of early and late-type morphologies based on their appearance on POSS plates, and that had not been observed in $\mathrm{H} \alpha$. One dwarf, NGC 5798, turned out to be a gas-rich normal dwarf irregular, and will not be discussed further here. It is important to note that environment was not a selection criteria, nor was the rate of ongoing star formation.

Direct imaging observations were made at Michigan-Dartmouth-MIT Observatory on the $1.3 \mathrm{~m}$ McGraw-Hill telescope. The data were taken on 10 - 12 April 1996 with a $1024 \mathrm{x} 1024$ thinned CCD. The pixel scale is $\sim 0.50 " /$ pix for this detector and telescope with the $\mathrm{f} / 7.5$ secondary, giving a field of view of $\sim 8.5^{\prime} \times 8.5^{\prime}$. Broadband $B$ and $R$ images were made using the MDM Schombert filter set. These filters quite closely approximate the wavelength coverage of the Kitt Peak National Observatory "Harris Set" of broadband filters, although the transmission efficiencies are lower. Narrowband $\mathrm{H} \alpha$ imaging was done using a set of extragalactic $\mathrm{H} \alpha$ interference filters. Typical integrations times were 20-30 minutes in $B, 10-15$ minutes in $R$, and 40-60 minutes in $\mathrm{H} \alpha$. Several Landolt standard fields (Landolt 1992) were observed each night in the broadband filters, and the spectroscopic standard Feige 34 was observed in $R$ and each $\mathrm{H} \alpha$ filter.

Initial data reduction was carried out in the standard way using IRAF $\dagger$. In order to obtain the $\mathrm{H} \alpha$ flux for each galaxy, the observations of the spectrophotometric standard Feige 34 were used. Feige 34 was observed under photometric conditions in both the $R$ and $\mathrm{H} \alpha$ filters. Full details of the data reduction can be found in Knezek, Sembach, \& Gallagher (1999).

\section{Results and Discussion}

We have determined the broadband and emission morphologies, as well as the broadband colors and surface brightness distributions for all the dwarfs in our initial sample. Figures 1 and 2 show all six galaxies. The top panel is the image of each galaxy in $\mathrm{H} \alpha$ emission. The bottom panel is the corresponding $R$ band data, shown as surface brightness contours. As noted in the previous section, NGC 5798 is a gas-rich dwarf irregular that was inadvertently included in the sample. Of the five remaining dwarfs, all have fairly smooth outer $R$ band isophotes, consistent with their "mixed" morphology and classification as a "transition" dwarf. Four of the five galaxies have very centrally-peak $\mathrm{H} \alpha$ emssion, with only very faint emission outside the central region. These galaxies are very similar to the 'dwarf amorphous nuclear starburst' (DANS) galaxies first described by Salzer, MacAlpine, \& Boroson (1989), but with blue luminosities that are 1-2 magnitudes fainter. The exception is NGC 3353, which has strong central emission, but also extended strong emission that shows a bar-like structure.

Table 1 shows a comparison of the derived properties for the five "transition" dwarfs. The four DANS galaxies all have strong $\mathrm{H} \alpha$ emission, relatively blue colors $(B-R \sim 0.95$ $1.25)$, are gas poor $\left(\mathrm{M}_{H I} / \mathrm{L}_{B} \lesssim 0.1\right)$, and are forming stars prodigiously, at a rate of $\sim 2$ $\mathrm{M}_{\text {sun }} / \mathrm{yr}$. NGC 3353 is even more extreme, with a very blue color $(B-R \sim 0.85)$, is relatively gas-rich $\left(\mathrm{M}_{H I} / \mathrm{L}_{B} \sim 0.3\right)$, and is forming stars at a rate that is three times higher than the four DANS galaxies. NGC 3353 is a well known BCD, and perhaps it represents an earlier phase of a starburst phenomenon. All five of these systems will exhaust their gas in less than a Hubble time if they continue to form stars at their current

$\dagger$ IRAF is distributed by the National Optical Astronomy Observatories, which are operated by the Association of Universities for Research in Astronomy, Inc., under contract to the National Science Foundation. 
Table 1. Derived Properties of the Dwarf "Transition" Galaxies

\begin{tabular}{lcccccc}
\hline Galaxy & $\mathrm{M}_{B}$ & $\mathrm{~B}-\mathrm{R}$ & $\mathrm{EW}(\mathrm{H} \alpha)$ & $\mathrm{M}_{H I} / \mathrm{L}_{B}$ & $\mathrm{SFR}(\mathrm{H} \alpha)$ & $\log$ \\
\hline & $(\mathrm{mag})$ & $(\mathrm{mag})$ & $($ angstrom $)$ & $\left(\mathrm{M}_{\text {sun }} / \mathrm{L}_{\text {sun }}\right)$ & $\left(\mathrm{M}_{\text {sun }} / \mathrm{yr}\right)$ & $\left(\mathrm{M}_{H \text { I }} / \mathrm{SFR}\right)$ \\
\hline IC 745 & -17.0 & 0.96 & 21 & $\ldots$ & 1.8 & 8.56 \\
NGC 3265 & -17.5 & 1.24 & 48 & 0.1 & 2.1 & 8.02 \\
NGC 3353 & -17.3 & 0.85 & $\ldots$ & 0.3 & 6.0 & 7.95 \\
NGC 3773 & -17.3 & 0.93 & 6.9 & 0.06 & 2.1 & 7.82 \\
NGC 3928 & -16.7 & 1.23 & 53 & 0.1 & 1.6 & 8.32 \\
\hline
\end{tabular}

rates. Furthermore, if they do exhaust their gas and begin to fade, they would evolve into dSph-like systems with a color gradient, which may be consistent with what is seen in our own, lower mass, LG dSphs, where some dwarfs appear to have significantly different star formation histories in their inner and outer regions (see Mateo 1998, and references therein).

Morphologically, the four DANS galaxies appear very similar to NGC 5253, the BCD that does not appear to be rotationally supported. However, they are forming stars at nearly an order of magnitude higher rate (NGC 5253 is forming stars at $\sim 0.35 \mathrm{M}_{\text {sun }} / \mathrm{yr}$, Marlowe et al. 1997). The outer regions of the DANS galaxies are currently not undergoing active star formation, and their colors are consistent with an intermediate age population ( $\sim 2-6$ Gyr; Dellenbusch et al. 2005). Furthermore, all five galaxies also turn out to share an environmental similarity to NGC 5253. They are all members of groups (IC 745, NGC 3265, NGC 3773) or clusters (NGC 3353, NGC 3928). Given that there was no selection criteria based on environment, and that many BCDs are found to be isolated, perhaps the starbursts in these systems have been induced in a fundamentally different way, e.g. through interactions with other galaxies. In particular, the very centralized nature of the star formation in the majority of the sample suggests that the gas may have been driven towards the center and induced a starburst that will quickly remove the gas and transform the systems into dE/dSphs (Moore, Lake, \& Katz 1998).

\section{Conclusions}

We have studied a sample of five "transition" dwarf galaxies with optical morphologies that have a mix of early and late-type dwarfs characteristics. We find that all five galaxies are forming stars at a very prodigious rate (1.5-6 $\left.\mathrm{M}_{\text {sun }} / \mathrm{yr}\right)$, and four of the five dwarfs have nearly all their star formation occuring in their centers. Their outer regions no longer appear to be forming stars, and have colors that are consistent with an intermediate age stellar population ( $\sim 2-6$ Gyr). None of the galaxies is isolated, and the centralized nature of the starburst suggests that the starburst may have been induced by interactions with other galaxies, rather than being internally triggered. If these galaxies continue to form stars at their current rates, they will exhaust their gas in much less than a Hubble time. We suggest that perhaps these systems are true "transition" dwarfs, caught in the act of transforming their gas into stars, and progenitors of more massive dEs/dSphs.

\section{Acknowledgements}

The $\mathrm{H} \alpha$ filter set used for this research was kindly loaned to us by Deidre Hunter. Rosa Gonzalez provided us with the scripts for the spectrophotometric data reduction, and Ricky Patterson offered many helpful comments on the surface photometry. In addition, we would like to thank the staff at the Michigan-Dartmouth-MIT Observatory for their assistance and support in this project. 

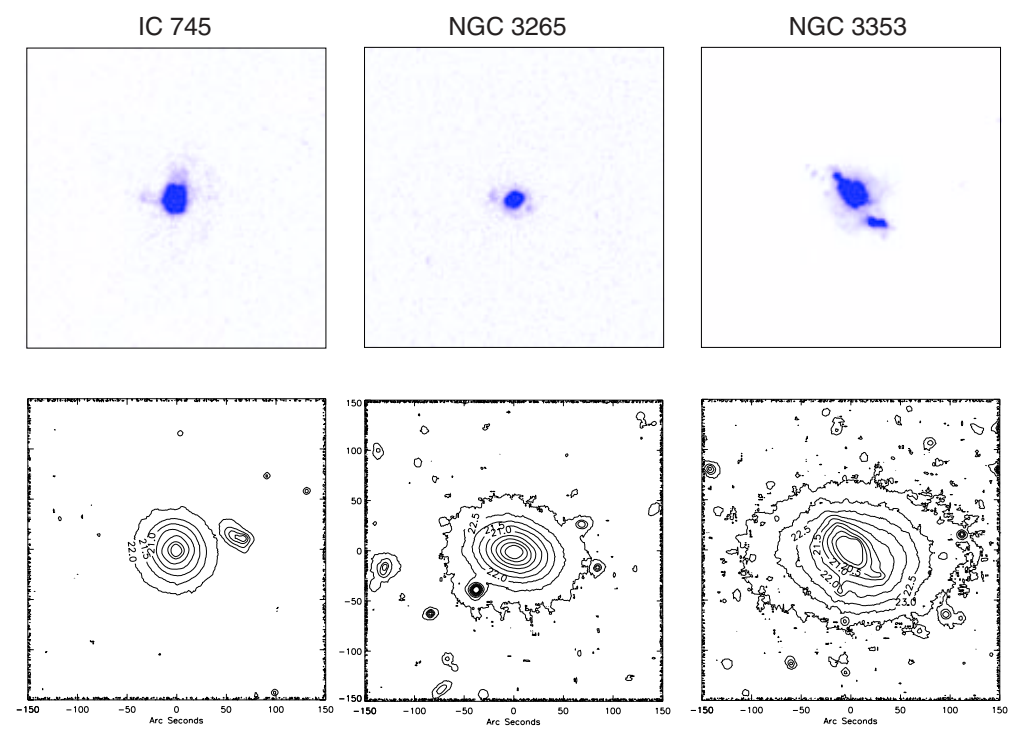

Figure 1. The upper panels show the $\mathrm{H} \alpha$ emission, while the lower panels indicate the R-band surface brightness contours in mag $\operatorname{arcsec}^{-2}$
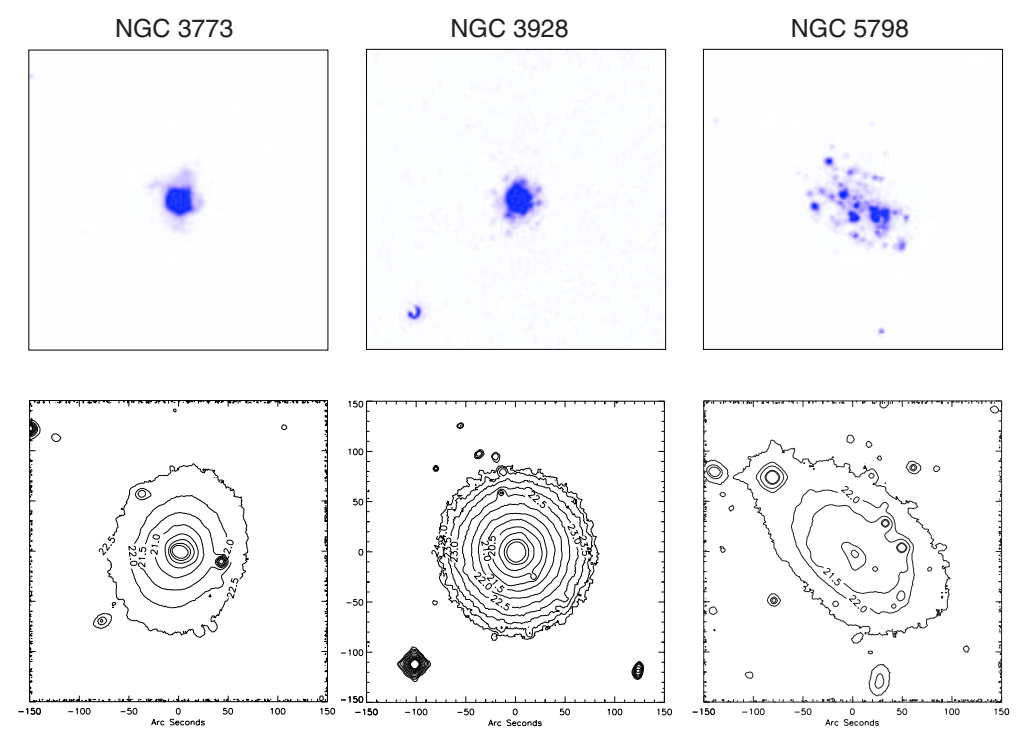

Figure 2. The upper panels show the $\mathrm{H} \alpha$ emission, while the lower panels indicate the R-band surface brightness contours in mag $\operatorname{arcsec}^{-2}$

\section{References}

Babul, A. \& Rees, M. J. 1992, MNRAS 255, 346

Binggeli, B. 1986, in Star Forming Dwarfs and Related Objects, eds. D. Kunth, T.X. Thuan, J.T.T. Thuan (Gif sur Yvette: Editiones Frontiéres), p. 52

Caldwell, N. \& Phillips, M.M. 1989, ApJ 338, 789

De Young, D. \& Heckman, T.F. 1994, ApJ 431, 598

Dekel, A. \& Silk, J. 1986, ApJ 303, 39 
Dellenbusch, K.D., Knezek, P.M., Gallagher, III, J.S. \& Sembach, K.R. 2005, in prep.

Grebel, E.K., Gallagher, III, J.S. \& Harbeck, D. 2003, $A J$ 125, 1926

Henriksen, M. \& Byrd, G. 1996, ApJ 459, 82

Knezek, P.M., Sembach, K.R. \& Gallagher, III, J.S. 1999, ApJ 514, 119

Kormendy, J. 1985, ApJ 295, 73

Landolt, A.U. 1992, $A J$ 104, 340

Lin, D.N.C. \& Faber, S.M. 1983, ApJ(Letters) 266, 21

Marlowe, A.T., Meurer, G.R., Heckman, T.M. \& Schommer, R. 1997, ApJS 112, 285

Mateo, M.L. 1998, ARA $\& A$ 36, 435

Mayer, L., Governato, F., Colpi, M., Moore, B., Quinn, T., Wadsley, J., Stadel, J. \& Lake, G. 2001a, ApJ 559, 754

Mayer, L., Governato, F., Colpi, M., Moore, B., Quinn, T., Wadsley, J., Stadel, J. \& Lake, G. 2001b, ApJ (Letters) 547, L123

Moore, B., Katz, N. \& Lake, G. 1996, ApJ 457, 455

Moore, B., Lake, G. \& Katz, N. 1998, ApJ 495, 139

Salzer, J.J., MacAlpine, G.M. \& Boroson, T.A. 1989, ApJS 70, 447

Sandage, A. \& Hoffman, G.L. 1991, ApJ(Letters) 379, L45

Sofue, Y. 1994, ApJ 423, 207

Spaans, M. \& Norman, C. 1997, ApJ 483, 87

Thuan, T. X. 1985, ApJ 299, 881

van Zee, L., Haynes, M.P. \& Salzer, J.J. 1997, AJ 114, 2479

van Zee, L., Salzer, J.J. \& Skillman, E.D. 2001, AJ 122, 121

van Zee, L., Skillman, E.D. \& Haynes, M.P. 2004, $A J$ 128, 121

\section{Discussion}

Drinkwater: Many of the dwarfs morphologically classified as dEs at the edge of the Fornax cluster have significant $\mathrm{H} \alpha$ emission (see poster by Michielsen et al.). How would these compare to the transition types you discussed? Their absolute magnitudes are around $M_{B}=-15$.

KNEZEK: These galaxies may well be members of the same family. The galaxies I showed are brighter $\left(M_{B} \approx-17\right)$, but we have not removed emission due to the starburst. If that is done, theirunderlying luminosities may be similar. Our sample might represent one extreme end of a family of galaxies that are modified due to their environment. It might be interesting to examine the relative environmental conditions of the galaxies in our sample (mostly in groups) with these dwarfs on the outskirts of Fornax.

CONSELICE: Does the fact that transition dwarfs are very rare imply a short life-time for this phase? Does this also imply that something must be removing the gas in these systems fairly quickly?

KNEZEK: Yes, the fact that these systems are rare implies that their life-times must be very short. 